\title{
Serum Brain-Derived Neurotrophic Factor Levels in Different Neurological Diseases
}

\author{
Mariacarla Ventriglia, ${ }^{1}$ Roberta Zanardini, ${ }^{2}$ \\ Cristina Bonomini, ${ }^{3}$ Orazio Zanetti, ${ }^{3}$ Daniele Volpe, ${ }^{4}$ Patrizio Pasqualetti, ${ }^{5}$ \\ Massimo Gennarelli, ${ }^{6,7}$ and Luisella Bocchio-Chiavetto ${ }^{2}$ \\ ${ }^{1}$ Department of Neuroscience, AFaR_Fatebenefratelli Hospital, Isola Tiberina 39, 00186 Rome, Italy \\ ${ }^{2}$ Neuropsychopharmacology Unit, IRCCS "S. Giovanni di Dio" Fatebenefratelli, Via Pilastroni 4, 25125 Brescia, Italy \\ ${ }^{3}$ Alzheimer's Disease Unit, IRCCS “S. Giovanni di Dio" Fatebenefratelli, Via Pilastroni 4, 25125 Brescia, Italy \\ ${ }^{4}$ Parkinson's Rehabilitation Unit, AFaR_Fatebenefratelli S. Raffaele Arcangelo Hospital, Madonna dell'Orto 3458, 30121 Venice, Italy \\ ${ }^{5}$ Medical Statistics \& Information Technology, AFaR_Fatebenefratelli Hospital, Isola Tiberina 39, 00186 Rome, Italy \\ ${ }^{6}$ Division of Biology and Genetics, Department of Molecular and Translational Medicine, University of Brescia, \\ Viale Europa 11, 25123 Brescia, Italy \\ ${ }^{7}$ Genetic Unit, IRCCS “S. Giovanni di Dio" Fatebenefratelli, Via Pilastroni 4, 25125 Brescia, Italy
}

Correspondence should be addressed to Luisella Bocchio-Chiavetto; lbocchio@fatebenefratelli.it

Received 30 April 2013; Revised 17 July 2013; Accepted 21 July 2013

Academic Editor: Elizabeta B. Mukaetova-Ladinska

Copyright (C) 2013 Mariacarla Ventriglia et al. This is an open access article distributed under the Creative Commons Attribution License, which permits unrestricted use, distribution, and reproduction in any medium, provided the original work is properly cited.

Consistent evidence indicates the involvement of the brain-derived neurotrophic factor (BDNF) in neurodegenerative disorders such as Alzheimer's disease (AD) and Parkinson's disease (PD). In the present study, we compared serum BDNF in 624 subjects: 266 patients affected by AD, 28 by frontotemporal dementia (FTD), 40 by Lewy body dementia (LBD), 91 by vascular dementia (VAD), 30 by PD, and 169 controls. Our results evidenced lower BDNF serum levels in AD, FTD, LBD, and VAD patients $(P<0.001)$ and a higher BDNF concentration in patients affected by $\mathrm{PD}(P=0.045)$. Analyses of effects of pharmacological treatments suggested significantly higher BDNF serum levels in patients taking mood stabilizers/antiepileptics $(P=0.009)$ and L-DOPA $(P<0.001)$ and significant reductions in patients taking benzodiazepines $(P=0.020)$. In conclusion, our results support the role of BDNF alterations in neurodegenerative mechanisms common to different forms of neurological disorders and underline the importance of including drug treatment in the analyses to avoid confounding effects.

\section{Introduction}

Neurological disorders have rapidly become a significant and growing problem, affecting more than 450 million individuals worldwide. The most common forms of neurodegenerative diseases include Alzheimer's disease (AD), Parkinson's disease (PD), frontotemporal dementia (FTD), vascular dementia (VAD), and dementia with Lewy bodies (LBD).

The identification of molecular dysregulations associated with the diagnosis of neurodegenerative disorders may provide important information for the clarification of the pathogenetic mechanisms and for the discovery of new biomarkers for an early, accurate, and differential diagnosis of these diseases [1].

Recently, there has been increasing evidence that alterations in the brain neurotrophic support and in particular in the brain-derived neurotrophic factor (BDNF) expression and signaling might contribute to neurodegeneration [2]. The BDNF is a member of the neurotrophin family of proteins that is not only important for the normal development of the peripheral and central nervous system but also plays a key role in neuronal survival and synaptic plasticity in the adult brain [3]. Altered functionality of BDNF has been observed in different neurodegenerative diseases $[4,5]$. A reduction of 
BDNF mRNA and protein expression has been consistently reported in multiple brain areas of $\mathrm{AD}$ postmortem and in the substantia nigra of PD patients [6,7]. Limited information is available for non-AD forms of dementia, although a neurotrophin decrease was observed in LBD but not in FTD patients $[8,9]$. Impairments in BDNF synthesis have been associated with the hallmarks of neurodegenerative pathogenesis [10]: a lack of BDNF has been selectively observed in neurons containing neurofibrillary tangles in $\mathrm{AD}$ [11], and a loss of BDNF production has been associated with mutations of alpha-synuclein in early-onset familial PD [12].

$\mathrm{BDNF}$ is also present in large quantities in blood platelets [13]. Despite the name "brain-derived," many peripheral sources, among them epithelial and vascular cells, muscle cells, macrophages, and leucocytes [14-16], may synthesize and release BDNF. The possibility of bidirectional transit of the neurotrophin across the blood-brain barrier is still an open area of investigation $[17,18]$. Nevertheless, evidence does suggest that serum BDNF levels may reflect what occurs in the brain since in recent years, the involvement of BDNF in the pathogenesis of several mental disorders has been corroborated by a series of biochemical studies. In particular, reduced BDNF serum concentrations were consistently observed in several adulthood psychiatric pathologies such as major depression disorder $[19,20]$. Furthermore, in healthy subjects, correlations were observed between serum BDNF levels and cerebral cortex integrity in neuroimaging studies [21] and in neuropsychological performances [22].

Peripheral BDNF levels have been analyzed in $\mathrm{AD}$ and PD patients reporting contrasting results. While some researchers have found a decrease in serum concentrations in $\mathrm{AD}$ and $\mathrm{PD}$ subjects [23-26], other studies have shown increased BDNF levels in patients in the early phases of $\mathrm{AD}$ $[23,27]$ and in patients with advanced AD [28]. One study found a decrease in serum BDNF in AD patients but did not show significant differences in VAD patients [29]. To date, no studies have investigated alterations of neurotrophin blood levels in other forms of neurodegenerative diseases, such as FTD or LBD. One possible explanation for the discrepancies in BDNF biochemical markers in the blood might be the possible effect of drug treatments in patients with neurodegenerative disorders. In fact, many drugs employed in the treatment of these pathologies, such as acetylcholinesterase inhibitors, anti-Parkinsonian agents, or antidepressants, may modulate BDNF expression [30-33].

In order to contribute in the clarification of the involvement of BDNF in neurodegenerative disease, replicating and extending previous findings, in this study we have compared BDNF serum levels in a large sample of patients affected by different neurological diseases and in a group of healthy participants. Moreover we have analyzed possible modifications induced by the most frequent drug treatments in neurodegenerative disorders to evaluate potential confounding effects on BDNF measures.

\section{Material and Methods}

2.1. Participants. The sample consisted of 624 participants: 169 controls, 266 AD, 28 FTD, 40 LBD, 91 VAD, and 30 idiopathic PD patients. Patients with AD, FTD, LBD, VAD, and control subjects were enrolled at the Alzheimer Unit of IRCCS-Fatebenefratelli (Brescia, Italy); PD patients were recruited at the Rehabilitation Unit, Hospital San Raffaele Arcangelo, Fatebenefratelli (Venezia, Italy). The study protocol was approved by the local ethics committees, and written informed consent was obtained from each individual prior to participation in the study. All patients were examined by neurologists with expertise in neurodegenerative diseases and met the internationally standardized criteria [34-39] for dementias or PD disorder. The severity of cognitive impairment was assessed by Mini-Mental State Examination scores (MMSE) [40]; staging in patients with PD was recorded with Hoehn and Yahr scores [41]. The majority of the patients involved in this study received pharmacological treatment for their illnesses and often took more than one medication simultaneously. Demographic and clinical features of the sample and the number of patients taking each of the more frequent classes of psychotropic medications (neuroleptics, benzodiazepines, antidepressants, mood stabilizers/antiepileptics, and cholinesterase inhibitors) are shown in Table 1. All PD patients received treatment with L-DOPA.

Control participants were recruited according to the following criteria: absence of alcohol, substance abuse, and relevant neurological diseases, MMSE scores of 27/30 or higher, and a negative personal and family history (first-degree relatives) of psychiatric DSM-IV Axis I disorders (confirmed with the Mini-International Neuropsychiatric Interview M.I.N.I).

2.2. Biochemical Investigation. Venous blood $(5 \mathrm{~mL})$ was collected from each patient in the morning (between 8 and 9 a.m.) after overnight fasting. Blood samples were collected into tubes without anticoagulant and allowed to clot at room temperature for $1 \mathrm{~h}$. Serum was separated by centrifuge at $1620 \mathrm{rcf}$ for $15 \mathrm{~min}$ and then stored at $-80^{\circ} \mathrm{C}$ until assay. BDNF levels were measured by the ELISA method using the human BDNF Quantikine kit (R\&D system, Minneapolis, USA), according to the manufacturer's instructions. BDNF content was expressed as the equivalent of the human recombinant protein. The detection limit was $20 \mathrm{pg} / \mathrm{mL}$, and data were expressed as ng of protein $/ \mathrm{mL}$ of serum. All samples and standards were measured in duplicate. Samples of patients with different diagnoses and control participants were analysed together in the ELISA templates, and possible variability between different assays was controlled using 4 samples of known BDNF concentration. The mean interassay precision, expressed as the coefficient of variation, was about $8 \%$.

\section{Statistical Analysis}

Statistical analyses included $T$-test, chi-squared test, analysis of variance (ANOVA) with post-hoc pairwise Bonferroni analysis, analysis of covariance (ANCOVA), and calculations of Pearson's linear correlation coefficients when appropriate. Significance for the results was set at $P<0.05$. SPSS, version 17.0 (http://www.spss.com/), was used for all statistical calculations. 
TABLE 1: Demographic, clinical features, and BDNF serum levels in different study groups.

\begin{tabular}{|c|c|c|c|c|c|c|}
\hline Diagnosis & CTRL & $\mathrm{AD}$ & FTD & LBD & VAD & $\mathrm{PD}$ \\
\hline Sample size & 169 & 266 & 28 & 40 & 91 & 30 \\
\hline \multicolumn{7}{|l|}{ Demographic variables } \\
\hline Gender (\% F) & $49 \%$ & $67 \%$ & $54 \%$ & $68 \%$ & $67 \%$ & $30 \%$ \\
\hline differences versus CTRL $P=$ & & $<0.001$ & 0.688 & 0.052 & 0.006 & 0.073 \\
\hline Age & $48.0 \pm 15.7$ & $80.1 \pm 7.1$ & $71.7 \pm 9.9$ & $79.2 \pm 5.6$ & $81.9 \pm 7.5$ & $67.6 \pm 8.4$ \\
\hline differences versus CTRL $P=$ & & $<0.001$ & $<0.001$ & $<0.001$ & $<0.001$ & $<0.001$ \\
\hline \multicolumn{7}{|l|}{ Clinical variables } \\
\hline MMSE score & $>27$ & $13.6 \pm 8.0$ & $16.1 \pm 6.6$ & $15.9 \pm 7.4$ & $14.8 \pm 8.0$ & $25.4 \pm 2.0$ \\
\hline HY score & & & & & & $3.58 \pm 0.5$ \\
\hline \multicolumn{7}{|l|}{ Pharmacological treatment } \\
\hline Neuroleptics $(-/+)^{*}$ & & $118 / 148$ & $14 / 14$ & $14 / 26$ & $44 / 47$ & $26 / 44$ \\
\hline Benzodiazepines $(-/+)$ & & $167 / 99$ & $17 / 11$ & $28 / 12$ & $58 / 33$ & $30 / 0$ \\
\hline Antidepressants $(-/+)$ & & $142 / 124$ & $12 / 16$ & $20 / 20$ & $27 / 64$ & $17 / 13$ \\
\hline Mood stabilizers antiepileptics $(-/+)$ & & $237 / 29$ & $20 / 8$ & $37 / 3$ & $74 / 17$ & $30 / 0$ \\
\hline Cholinesterase inhibitors $(-/+)$ & & $193 / 73$ & $27 / 1$ & $21 / 19$ & $87 / 4$ & $30 / 0$ \\
\hline L-DOPA $(-/+)$ & & $266 / 0$ & $28 / 0$ & $40 / 0$ & $91 / 0$ & $0 / 30$ \\
\hline \multicolumn{7}{|l|}{ Serum BDNF } \\
\hline $\mathrm{ng} / \mathrm{mL}($ mean $\pm \mathrm{SD})$ & $39.89 \pm 9.48$ & $33.16 \pm 12.4$ & $31.19 \pm 9.68$ & $31.71 \pm 11.6$ & $33.06 \pm 12.4$ & $46.13 \pm 15.3$ \\
\hline
\end{tabular}

$*(-/+)$ : not treated/treated.

\section{Results}

Demographic characteristics for controls and patients are reported in Table 1. No effects of age and sex variables on serum BDNF were observed in the control group: despite a large age range $(\min =19, \max =88, \mathrm{SD}=15.7)$ and a balanced sex distribution ( $49 \% \mathrm{~F}, 51 \% \mathrm{M}$ ), only $0.3 \%$ of BDNF variability was accounted for by age $(P=0.316)$ and $1.3 \%$ by $\operatorname{sex}(P=0.137)$. However, significant differences were observed in age and sex distributions between patients and controls in our sample (Table 1). Thus, possible confounding effects of these variables were controlled by including them as covariates in all statistical analyses. BDNF serum levels (Table 1, Figure 1) in the controls and in the patient groups were compared by one-way analyses of variance (ANOVA), and significant differences were found $(\mathrm{F}(5,621)=17.41, P<$ 0.001). Post-hoc analyses, using Bonferroni correction, found significant decreases in serum BDNF levels in AD, FTD, LBD and VAD patients compared with controls $(P<0.001)$. On the contrary, significant increases in serum BDNF levels were observed for PD patients $(P=0.045)$. All differences remained statistically significant after ANOVA analyses for sex and age variables.

No correlation between BDNF serum levels and cognitive impairments, as measured by scores on the MMSE, were observed in any dementia groups $(P>0.20)$ (PD patients are not included in this analysis). Analogously, we found no correlation between BDNF serum levels and PD symptoms, as measured with the HY scale $(P=0.45)$.

Analyses of potential modulatory effects of pharmacological treatments (Table 2, Figure 2) on BDNF levels in the whole sample of patients taking various common psychiatric medications revealed higher BDNF serum levels in patients treated with mood stabilizers/antiepileptics $(P=0.009)$ and
TABLE 2: BDNF serum levels (mean \pm SD) in patients taking $(+)$ and not taking (-) different psychotropic drugs.

\begin{tabular}{lcc}
\hline Pharmacological treatment & $N$ & BDNF ng/mL \\
\hline Neuroleptics (+) & 239 & $33.06 \pm 10.65$ \\
Neuroleptics (-) & 216 & $34.50 \pm 12.15$ \\
Benzodiazepines (+) & 155 & $32.02 \pm 9.81$ \\
Benzodiazepines (-) & 300 & $34.64 \pm 12.06$ \\
Antidepressants (+) & 237 & $33.14 \pm 11.05$ \\
Antidepressants (-) & 218 & $34.40 \pm 11.76$ \\
Mood stabilizers antiepileptics (+) & 57 & $37.43 \pm 12.32$ \\
Mood stabilizers antiepileptics (-) & 398 & $33.21 \pm 11.18$ \\
Cholinesterase inhibitors (+) & 97 & $32.36 \pm 10.64$ \\
Cholinesterase inhibitors (-) & 358 & $34.12 \pm 11.58$ \\
L-DOPA (+) & 30 & $46.13 \pm 15.30$ \\
L-DOPA (-) & 425 & $32.87 \pm 10.56$ \\
\hline
\end{tabular}

L-DOPA $(P<0.001)$ and significant reductions in neurotrophin concentrations in patients treated with benzodiazepines $(P=0.020)$. Differences in BDNF serum levels in demented patients toward controls remained significant after correction for the presence of pharmacological treatment with mood stabilizers/antiepileptics and benzodiazepines. Since all PD patients were administered L-DOPA and none of the other patients were taking such drug, the confounding effect could not be solved, and we could not assess whether the BDNF increase in PD patients was due to L-DOPA medication. 


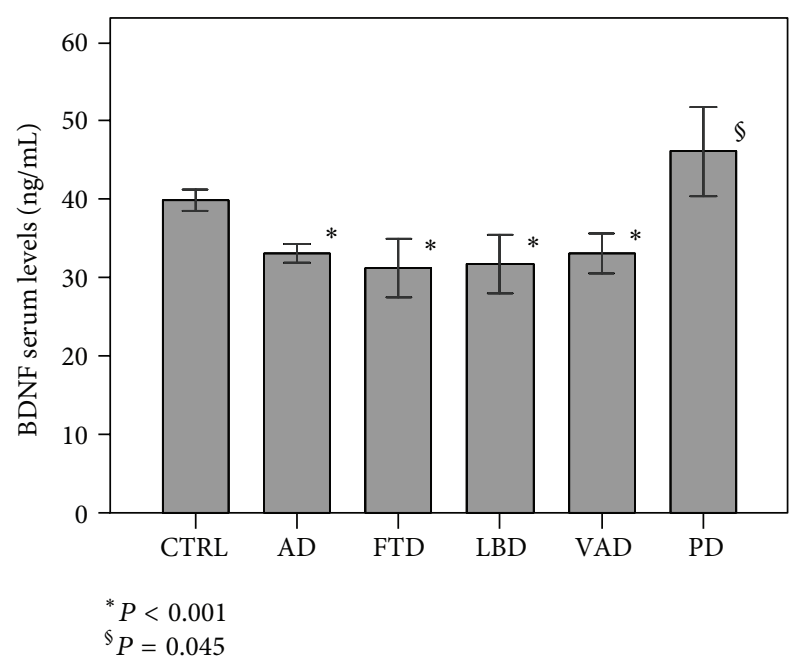

FIgURE 1: BDNF serum levels (mean, 95\% CI) in patient and control samples ( $P$ values are referred to comparisons versus controls).

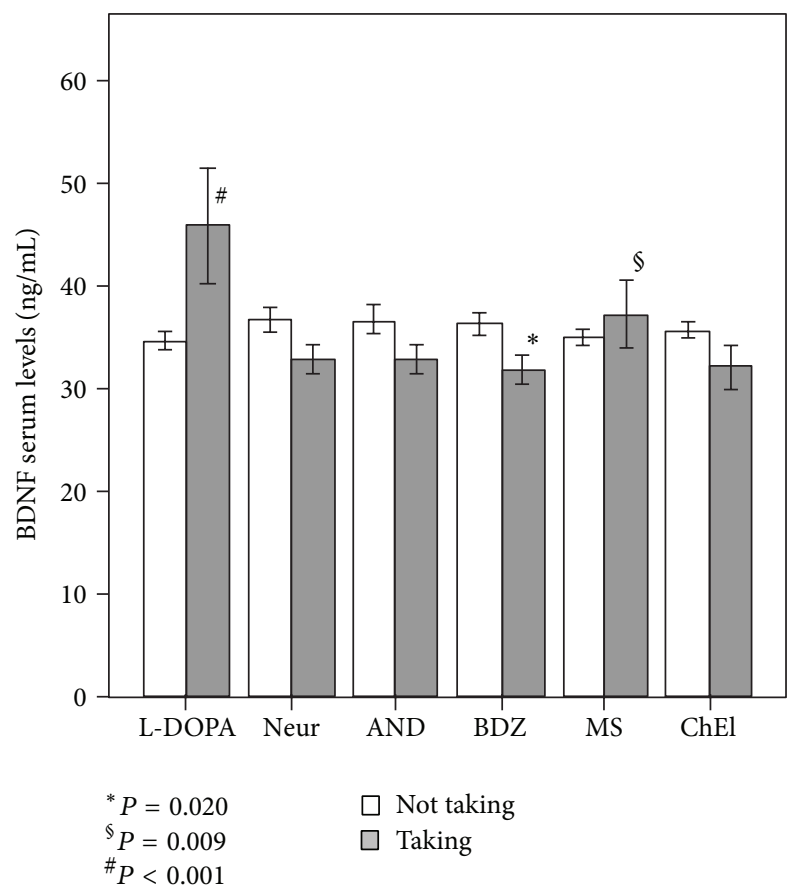

Figure 2: BDNF serum levels (mean, 95\% CI) in patients taking (grey) and not taking (white) different psychotropic drugs (neuroleptics: Neur, benzodiazepines: BDZ, antidepressants: AND, mood stabilizers/antiepileptics: MS, cholinesterase inhibitors: ChEI and LDOPA).

\section{Discussion}

The principal objective of our work was to evaluate putative alterations of BDNF serum levels in patients affected by neurodegenerative disorders and possible applications of the serum dosage as a biochemical marker for differential diagnosis. Our results showed a decrease in BDNF serum levels common to the different forms of dementias and an increase in the neutrophin content in patients affected by PD.
In accordance with our results, decreased serum concentrations of BDNF have been described in patients affected by $\mathrm{AD}[23,24,29,42]$, although some studies have reported no differences $[28,42]$ or even increases in neurotrophin levels [27]. These discrepancies might be explained, at least in part, by differences in the severity of the cognitive impairments associated with the different $\mathrm{AD}$ samples. In fact, increased serum BDNF levels have been observed in patients with MCI and early $\mathrm{AD}[27,42]$, supporting a possible compensatory augmentation of neurotrophin synthesis in the earliest stages of $\mathrm{AD}$ progression. Our sample was instead composed of $\mathrm{AD}$ patients with a moderate/severe degree of cognitive impairment (MMSE 13.6 \pm 8.0 ), and no associations were observed between illness severity and BDNF serum levels in our sample. We observed for the first time lower serum BDNF levels in VAD, LBD, and FTD patients, indicating a generalized reduction of the neurotrophin in dementia. Our data on VAD contrast with negative findings reported by Yasutake and colleagues [29]. Again, in this case, the inconsistency in reported results might be ascribed to the advanced cognitive impairment of our sample (MMSE $14.8 \pm 8.0$ ).

Overall, the results of our study limited the usefulness of BDNF as a biomarker for specific forms of dementias and, instead, support the involvement of BDNF in common pathogenetic mechanisms in all cognitive disorders analyzed. The BDNF pathway has been consistently linked to core pathological features of amyloidosis and tauopathies because this trophic factor is able to regulate the production and the neurotoxic effects of amyloid b42 and Tau protein hyperphosphorylation $[43,44]$. Furthermore, BDNF can elicit neuroprotective effects on neurons after vascular damage [45]. In general, BDNF seems to be essential to the survival and the functioning of mature, fully developed neurons in the adult brain, and perturbations in neurotrophin expression and trafficking have been associated with a wide spectrum of neurological and psychiatric disorders [46]. Interestingly, reduced serum BDNF levels have also been associated with worse cognitive performance in healthy elderly subjects [22], suggesting that variations in BDNF concentrations might also indicate a progressive shift toward a neurodegenerative state in the healthy elderly [47]. Finally, we found an increase in serum BDNF levels in PD patients, in partial disagreement with a recent study reporting decreased levels of BDNF in patients in an early stage of PD disease and with a moderate severity of motor function impairment [26]. PD patients in our study had severe motor impairments. Scalzo and colleagues also reported higher serum BDNF levels in patients in advanced stages of the disease. Moreover, the observed increase in BDNF in PD patients in our study could be explained by compensatory effects of pharmacological treatment with L-DOPA, whose increase might be viewed as a neuroprotective mechanism. Treatment with LDOPA induces BDNF release from corticostriatal fibers, and this mechanism consecutively enhances the expression of D3 receptors and maximizes the beneficial symptomatic effects of treatment [48].

These observations underline the necessity of controlling for the presence of drug treatment in biochemical studies of serum BDNF. Patients with dementias and PD are 
treated with a plethora of psychiatric drugs, ranging from cholinesterase inhibitors and L-DOPA used to treat core symptoms to antidepressants, neuroleptics, mood stabilizers and benzodiazepines used to manage the behavioral and psychotic symptoms that often accompany these diseases [49]. Numerous clinical studies evidenced the effect of medication, including antidepressants, mood stabilizers and atypical antipsychotics $[32,50,51]$ or lithium and cholinesterase inhibitors $[23,30]$ on the BDNF concentrations in neuropsychiatric diseases. In this study, significantly lower concentrations of BDNF were observed in patients treated with benzodiazepines $(P=0.020)$. The relationship between BDNF levels and benzodiazepines has been already investigated in other neuropsychiatric disorders. Diazepam was reported to reduce levels of BDNF [52] in animals, and, more recently, decreased levels of serum BDNF have been observed in schizophrenia patients with catatonia treated with lorazepam [53]. This association would seem to be linked to the known effect of BDNF on the GABA neurotransmitter [54-56] responsible for the sedative and anxiolytic effects of benzodiazepines. Moreover, an increase in BDNF levels was observed in patients taking a mood stabilizer that is in accordance with the hypothesis [57] that these drugs play an essential role in regulating BDNF expression. However, results obtained from studies of peripheral BDNF serum changes induced by these therapeutic drugs are not always consistent $[58,59]$ and may depend not only on the pharmacological characteristics of the drug but also on length of treatment [60].

Except for L-DOPA, for which the effect couldn't be discriminated, the differences in BDNF levels in our work remained significant for all dementias after correcting for the effect of various pharmacological treatments.

This study presents some limitations related mainly to the different size of the sample in fact for some pathologies under study the number of cases analyzed was lower than others. In addition, many of the patients recruited were in multitherapy, and this is certainly a further confounding factor for the interpretation of results.

\section{Conclusions}

In conclusion, our findings support the hypothesis that BDNF alterations are involved in neurodegenerative mechanisms and suggest that low BDNF levels are nonspecific markers of neurodegeneration common to several cognitive disorders. Our study also underlines the importance to control for treatment with psychotropic drugs in all analyses of BDNF to avoid confounding effects. Indeed, our results provide further information about the involvement of BDNF in dementias and in PD for the implementation of new therapeutic strategies.

\section{Acknowledgments}

This research was supported by grants from the Italian Ministry of Health and Fondazione Cariplo. The authors declare no conflict of interest.

\section{References}

[1] J. Genius, H. Klafki, J. Benninghoff, H. Esselmann, and J. Wiltfang, "Current application of neurochemical biomarkers in the prediction and differential diagnosis of Alzheimer's disease and other neurodegenerative dementias," European Archives of Psychiatry and Clinical Neuroscience, vol. 262, no. 2, pp. 71-77, 2012.

[2] S. Balaratnasingam and A. Janca, "Brain derived neurotrophic factor: a novel neurotrophin involved in psychiatric and neurological disorders," Pharmacology and Therapeutics, vol. 134, no. 1, pp. 116-124, 2012.

[3] O. Arancio and M. V. Chao, "Neurotrophins, synaptic plasticity and dementia," Current Opinion in Neurobiology, vol. 17, no. 3, pp. 325-330, 2007.

[4] C. Zuccato and E. Cattaneo, "Brain-derived neurotrophic factor in neurodegenerative diseases," Nature Reviews Neurology, vol. 5, no. 6, pp. 311-322, 2009.

[5] B. S. Diniz and A. L. Teixeira, "Brain-derived neurotrophic factor and Alzheimer's disease: physiopathology and beyond," NeuroMolecular Medicine, vol. 13, no. 4, pp. 217-222, 2011.

[6] M. G. Murer, Q. Yan, and R. Raisman-Vozari, "Brain-derived neurotrophic factor in the control human brain, and in Alzheimer's disease and Parkinson's disease," Progress in Neurobiology, vol. 63, no. 1, pp. 71-124, 2001.

[7] S. Peng, J. Wuu, E. J. Mufson, and M. Fahnestock, "Precursor form of brain-derived neurotrophic factor and mature brainderived neurotrophic factor are decreased in the pre-clinical stages of Alzheimer's disease," Journal of Neurochemistry, vol. 93, no. 6, pp. 1412-1421, 2005.

[8] K. Imamura, N. Hishikawa, K. Ono et al., "Cytokine production of activated microglia and decrease in neurotrophic factors of neurons in the hippocampus of Lewy body disease brains," Acta Neuropathologica, vol. 109, no. 2, pp. 141-150, 2005.

[9] I. Ferrer, C. Marín, M. J. Rey, and T. Ribalta, "Brain-derived neurotrophic factor in patients with frontotemporal dementia," Neuroscience Letters, vol. 279, no. 1, pp. 33-36, 2000.

[10] M. P. Mattson, "Glutamate and neurotrophic factors in neuronal plasticity and disease," Annals of the New York Academy of Sciences, vol. 1144, pp. 97-112, 2008.

[11] M. G. Murer, F. Boissiere, Q. Yan et al., "An immunohistochemical study of the distribution of brain-derived neurotrophic factor in the adult human brain, with particular reference to Alzheimer's disease," Neuroscience, vol. 88, no. 4, pp. 1015-1032, 1999.

[12] R. Kohno, H. Sawada, Y. Kawamoto, K. Uemura, H. Shibasaki, and S. Shimohama, "BDNF is induced by wild-type $\alpha$-synuclein but not by the two mutants, A30P or A53T, in glioma cell line," Biochemical and Biophysical Research Communications, vol. 318, no. 1, pp. 113-118, 2004.

[13] H. Fujimura, C. A. Altar, R. Chen et al., "Brain-derived neurotrophic factor is stored in human platelets and released by agonist stimulation," Thrombosis and Haemostasis, vol. 87, no. 4, pp. 728-734, 2002.

[14] A. Gielen, M. Khademi, S. Muhallab, T. Olsson, and F. Piehl, "Increased brain-derived neurotrophic factor expression in white blood cells of relapsing-remitting multiple sclerosis patients," Scandinavian Journal of Immunology, vol. 57, no. 5, pp. 493-497, 2003.

[15] T. Nakahashi, H. Fujimura, C. A. Altar et al., "Vascular endothelial cells synthesize and secrete brain-derived neurotrophic factor," FEBS Letters, vol. 470, no. 2, pp. 113-117, 2000. 
[16] A. Cattaneo, L. Bocchio-Chiavetto, R. Zanardini, E. Milanesi, A. Placentino, and M. Gennarelli, "Reduced peripheral brainderived neurotrophic factor mRNA levels are normalized by antidepressant treatment," International Journal of Neuropsychopharmacology, vol. 13, no. 1, pp. 103-108, 2010.

[17] W. Pan, W. A. Banks, M. B. Fasold, J. Bluth, and A. J. Kastin, "Transport of brain-derived neurotrophic factor across the blood-brain barrier," Neuropharmacology, vol. 37, no. 12, pp. 1553-1561, 1998.

[18] J. F. Poduslo and G. L. Curran, "Permeability at the blood-brain and blood-nerve barriers of the neurotrophic factors: NGF, CNTF, NT-3, BDNF," Molecular Brain Research, vol. 36, no. 2, pp. 280-286, 1996.

[19] L. Bocchio-Chiavetto, V. Bagnardi, R. Zanardini et al., "Serum and plasma BDNF levels in major depression: a replication study and meta-analyses," World Journal of Biological Psychiatry, vol. 11, no. 6, pp. 763-773, 2010.

[20] E. Castrén and T. Rantamäki, "The role of BDNF and its receptors in depression and antidepressant drug action: reactivation of developmental plasticity," Developmental Neurobiology, vol. 70, no. 5, pp. 289-297, 2010.

[21] U. E. Lang, R. Hellweg, F. Seifert, F. Schubert, and J. Gallinat, "Correlation between serum brain-derived neurotrophic factor level and an in vivo marker of cortical integrity," Biological Psychiatry, vol. 62, no. 5, pp. 530-535, 2007.

[22] J. Gunstad, A. Benitez, J. Smith et al., "Serum brain-derived neurotrophic factor is associated with cognitive function in healthy older adults," Journal of Geriatric Psychiatry and Neurology, vol. 21, no. 3, pp. 166-170, 2008.

[23] C. Laske, E. Stransky, T. Leyhe et al., "BDNF serum and CSF concentrations in Alzheimer's disease, normal pressure hydrocephalus and healthy controls," Journal of Psychiatric Research, vol. 41, no. 5, pp. 387-394, 2007.

[24] O. V. Forlenza, B. S. Diniz, A. L. Teixeira et al., "Effect of brainderived neurotrophic factor Val66Met polymorphism and serum levels on the progression of mild cognitive impairment," World Journal of Biological Psychiatry, vol. 11, no. 6, pp. 774-780, 2010.

[25] G. L. Jung, S. S. Bae, S. Y. Young et al., "Decreased serum brain-derived neurotrophic factor levels in elderly Korean with dementia," Psychiatry Investigation, vol. 6, no. 4, pp. 299-305, 2009.

[26] P. Scalzo, A. Kümmer, T. L. Bretas, F. Cardoso, and A. L. Teixeira, "Serum levels of brain-derived neurotrophic factor correlate with motor impairment in Parkinson's disease," Journal of Neurology, vol. 257, no. 4, pp. 540-545, 2010.

[27] F. Angelucci, A. Oliviero, F. Pilato et al., "Transcranial magnetic stimulation and BDNF plasma levels in amyotrophic lateral sclerosis," NeuroReport, vol. 15, no. 4, pp. 717-720, 2004.

[28] S. E. O'Bryant, V. L. Hobson, J. R. Hall et al., "Serum brainderived neurotrophic factor levels are specifically associated with memory performance among Alzheimer's disease cases," Dementia and Geriatric Cognitive Disorders, vol. 31, no. 1, pp. 31-36, 2011.

[29] C. Yasutake, K. Kuroda, T. Yanagawa, T. Okamura, and H. Yoneda, "Serum BDNF, TNF- $\alpha$ and IL- $1 \beta$ levels in dementia patients: comparison between Alzheimer's disease and vascular dementia," European Archives of Psychiatry and Clinical Neuroscience, vol. 256, no. 7, pp. 402-406, 2006.

[30] T. Leyhe, G. W. Eschweiler, E. Stransky et al., "Increase of BDNF serum concentration in lithium treated patients with early alzheimer's disease," Journal of Alzheimer's Disease, vol. 16, no. 3, pp. 649-656, 2009.

[31] X. Zhang, P. E. Andren, and P. Svenningsson, "Repeated 1DOPA treatment increases c-fos and BDNF mRNAs in the subthalamic nucleus in the 6-OHDA rat model of Parkinson's disease," Brain Research, vol. 1095, no. 1, pp. 207-210, 2006.

[32] S. Sen, R. Duman, and G. Sanacora, "Serum brain-derived neurotrophic factor, depression, and antidepressant medications: meta-analyses and Implications," Biological Psychiatry, vol. 64, no. 6, pp. 527-532, 2008.

[33] V. Ricci, M. Pomponi, G. Martinotti et al., "Antidepressant treatment restores brain-derived neurotrophic factor serum levels and ameliorates motor function in parkinson disease patients," Journal of Clinical Psychopharmacology, vol. 30, no. 6, pp. 751-753, 2010.

[34] G. McKhann, D. Drachman, M. Folstein, R. Katzman, D. Price, and E. M. Stadlan, "Clinical diagnosis of Alzheimer's disease: report of the NINCDS-ADRDA work group under the auspices of Department of Health and Human Services Task Force on Alzheimer's disease," Neurology, vol. 34, no. 7, pp. 939-944, 1984.

[35] American Psychiatric Association, Diagnostic and Statistical Manual of Mental Disorders, Text Revision, Washington, DC, USA, 4th edition, 2000.

[36] D. Neary, J. S. Snowden, L. Gustafson et al., "Frontotemporal lobar degeneration: a consensus on clinical diagnostic criteria," Neurology, vol. 51, no. 6, pp. 1546-1554, 1998.

[37] I. G. McKeith, D. W. Dickson, J. Lowe et al., "Diagnosis and management of dementia with Lewy bodies: third report of the DLB consortium," Neurology, vol. 65, no. 12, pp. 1863-1872, 2005.

[38] G. C. Román, T. K. Tatemichi, T. Erkinjuntti et al., "Vascular dementia: diagnostic criteria for research studies: report of the NINDS-AIREN International Workshop," Neurology, vol. 43, no. 2, pp. 250-260, 1993.

[39] M. Emre, D. Aarsland, R. Brown et al., "Clinical diagnostic criteria for dementia associated with Parkinson's disease," Movement Disorders, vol. 22, no. 12, pp. 1689-1707, 2007.

[40] M. F. Folstein, S. E. Folstein, and P. R. McHugh, “"Mini-mental state": a practical method for grading the cognitive state of patients for the clinician," Journal of Psychiatric Research, vol. 12, no. 3, pp. 189-198, 1975.

[41] M. M. Hoehn and M. D. Yahr, "Parkinsonism: onset, progression and mortality," Neurology, vol. 17, no. 5, pp. 427-442, 1967.

[42] C. Laske, E. Stransky, T. Leyhe et al., "Decreased brain-derived neurotrophic factor (BDNF)- and $\beta$-thromboglobulin $(\beta$-TG)blood levels in Alzheimer's disease," Thrombosis and Haemostasis, vol. 96, no. 1, pp. 102-103, 2006.

[43] M. Rohe, M. Synowitz, R. Glass, S. M. Paul, A. Nykjaer, and T. E. Willnow, "Brain-derived neurotrophic factor reduces amyloidogenic processing through control of SORLA gene expression," The Journal of Neuroscience, vol. 29, no. 49, pp. 15472-15478, 2009.

[44] E. Elliott, R. Atlas, A. Lange, and I. Ginzburg, "Brain-derived neurotrophic factor induces a rapid dephosphorylation of tau protein through a PI-3Kinase signalling mechanism," European Journal of Neuroscience, vol. 22, no. 5, pp. 1081-1089, 2005.

[45] I. Ferrer, J. Krupinski, E. Goutan, E. Martí, S. Ambrosio, and E. Arenas, "Brain-derived neurotrophic factor reduces cortical cell death by ischemia after middle cerebral artery occlusion in the rat," Acta Neuropathologica, vol. 101, no. 3, pp. 229-238, 2001. 
[46] A. H. Nagahara and M. H. Tuszynski, "Potential therapeutic uses of BDNF in neurological and psychiatric disorders," Nature Reviews Drug Discovery, vol. 10, no. 3, pp. 209-219, 2011.

[47] A. L. Teixeira, I. G. Barbosa, B. S. Diniz, and A. Kummer, "Circulating levels of brain-derived neurotrophic factor: correlation with mood, cognition and motor function," Biomarkers in Medicine, vol. 4, no. 6, pp. 871-887, 2010.

[48] R. M. Kostrzewa, P. Nowak, J. P. Kostrzewa, R. A. Kostrzewa, and R. Brus, "Peculiarities of L-DOPA treatment of Parkinson's disease," Amino Acids, vol. 28, no. 2, pp. 157-164, 2005.

[49] C. W. Zhu, E. E. Livote, K. Kahle-Wrobleski et al., "Utilization of antihypertensives, antidepressants, antipsychotics, and hormones in Alzheimer disease," Alzheimer Disease and Associated Disorders, vol. 25, no. 2, pp. 144-148, 2011.

[50] B. Chen, D. Dowlatshahi, G. M. MacQueen, J.-F. Wang, and L. T. Young, "Increased hippocampal BDNF immunoreactivity in subjects treated with antidepressant medication," Biological Psychiatry, vol. 50, no. 4, pp. 260-265, 2001.

[51] E. Shimizu, K. Hashimoto, N. Okamura et al., "Alterations of serum levels of brain-derived neurotrophic factor (BDNF) in depressed patients with or without antidepressants," Biological Psychiatry, vol. 54, no. 1, pp. 70-75, 2003.

[52] L. Huopaniemi, R. Keist, A. Randolph, U. Certa, and U. Rudolph, "Diazepam-induced adaptive plasticity revealed by $\alpha 1$ GABAA receptor-specific expression profiling," Journal of Neurochemistry, vol. 88, no. 5, pp. 1059-1067, 2004.

[53] T.-L. Huang and Y.-Y. Hung, "Lorazepam reduces the serum brain-derived neurotrophic factor level in schizophrenia patients with catatonia," Progress in Neuro-Psychopharmacology and Biological Psychiatry, vol. 33, no. 1, pp. 158-159, 2009.

[54] N. Canas, I. T. Pereira, J. A. Ribeiro, and A. M. Sebastião, "Brainderived neurotrophic factor facilitates glutamate and inhibits GABA release from hippocampal synaptosomes through different mechanisms," Brain Research, vol. 1016, no. 1, pp. 72-78, 2004.

[55] T. Matsumoto, T. Numakawa, D. Yokomaku et al., "Brainderived neurotrophic factor-induced potentiation of glutamate and GABA release: different dependency on signaling pathways and neuronal activity," Molecular and Cellular Neuroscience, vol. 31, no. 1, pp. 70-84, 2006.

[56] B. Singh, C. Henneberger, D. Betances et al., "Altered balance of glutamatergic/GABAergic synaptic input and associated changes in dendrite morphology after BDNF expression in BDNF-deficient hippocampal neurons," The Journal of Neuroscience, vol. 26, no. 27, pp. 7189-7200, 2006.

[57] S. Nishino, K. Ohtomo, Y. Numata, T. Sato, N. Nakahata, and M. Kurita, "Divergent effects of lithium and sodium valproate on brain-derived neurotrophic factor (BDNF) production in human astrocytoma cells at therapeutic concentrations," Progress in Neuro-Psychopharmacology and Biological Psychiatry, vol. 39, no. 1, pp. 17-22, 2012.

[58] J. Haymond and M. H. H. Ensom, "Does valproic acid warrant therapeutic drug monitoring in bipolar affective disorder?" Therapeutic Drug Monitoring, vol. 32, no. 1, pp. 19-29, 2010.

[59] L. K. Jornada, M. Moretti, S. S. Valvassori et al., "Effects of mood stabilizers on hippocampus and amygdala BDNF levels in an animal model of mania induced by ouabain," Journal of Psychiatric Research, vol. 44, no. 8, pp. 506-510, 2010.

[60] F. Matrisciano, S. Bonaccorso, A. Ricciardi et al., "Changes in BDNF serum levels in patients with major depression disorder (MDD) after 6 months treatment with sertraline, escitalopram, or venlafaxine," Journal of Psychiatric Research, vol. 43, no. 3, pp. 247-254, 2009. 


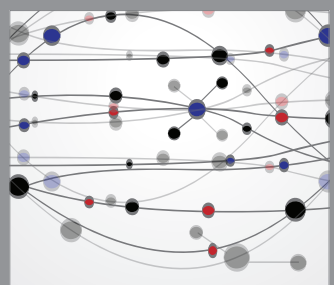

The Scientific World Journal
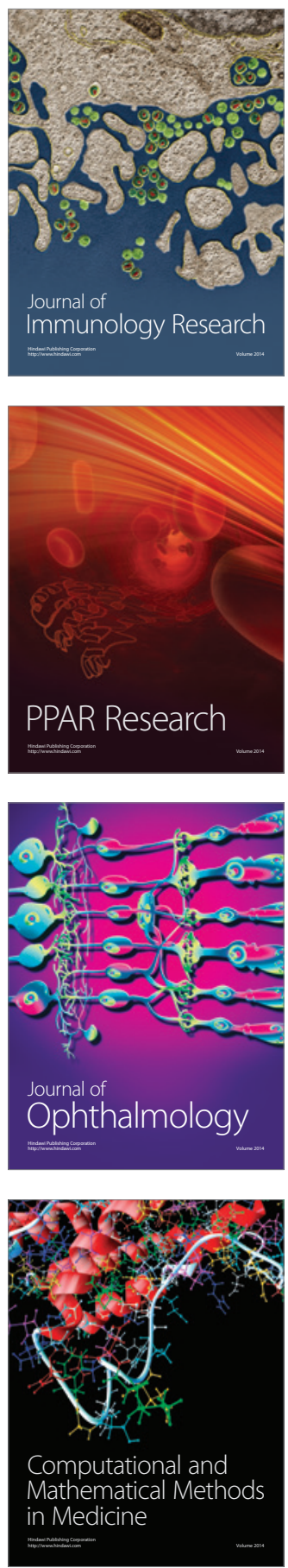

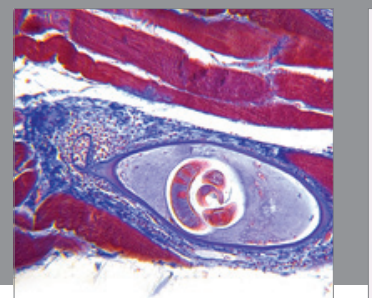

Gastroenterology

Research and Practice
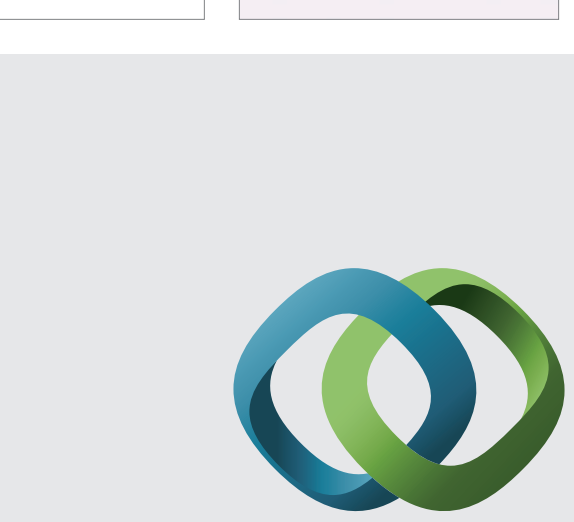

\section{Hindawi}

Submit your manuscripts at

http://www.hindawi.com
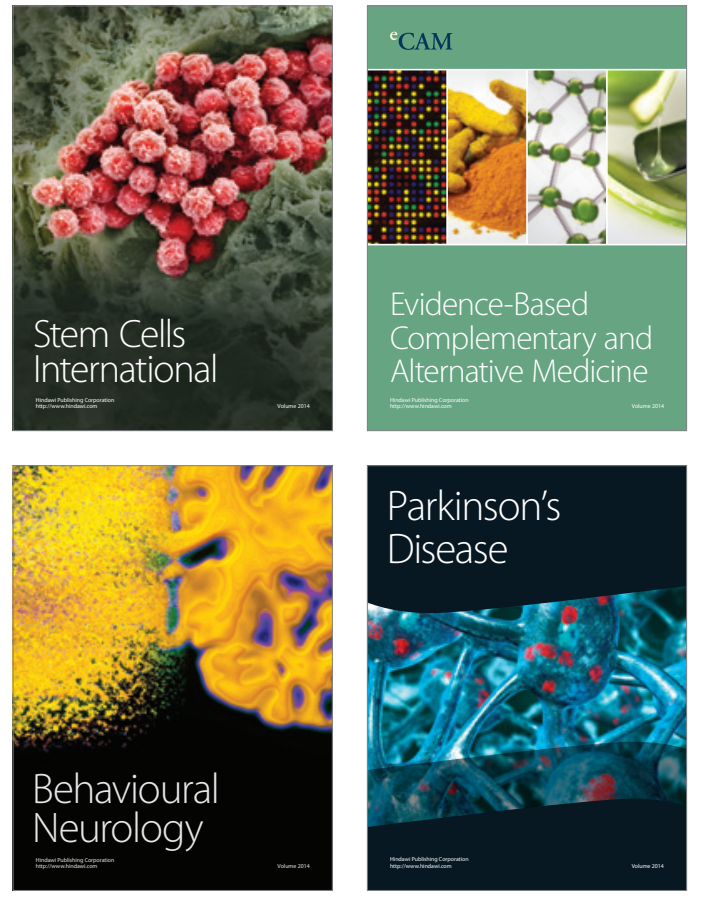
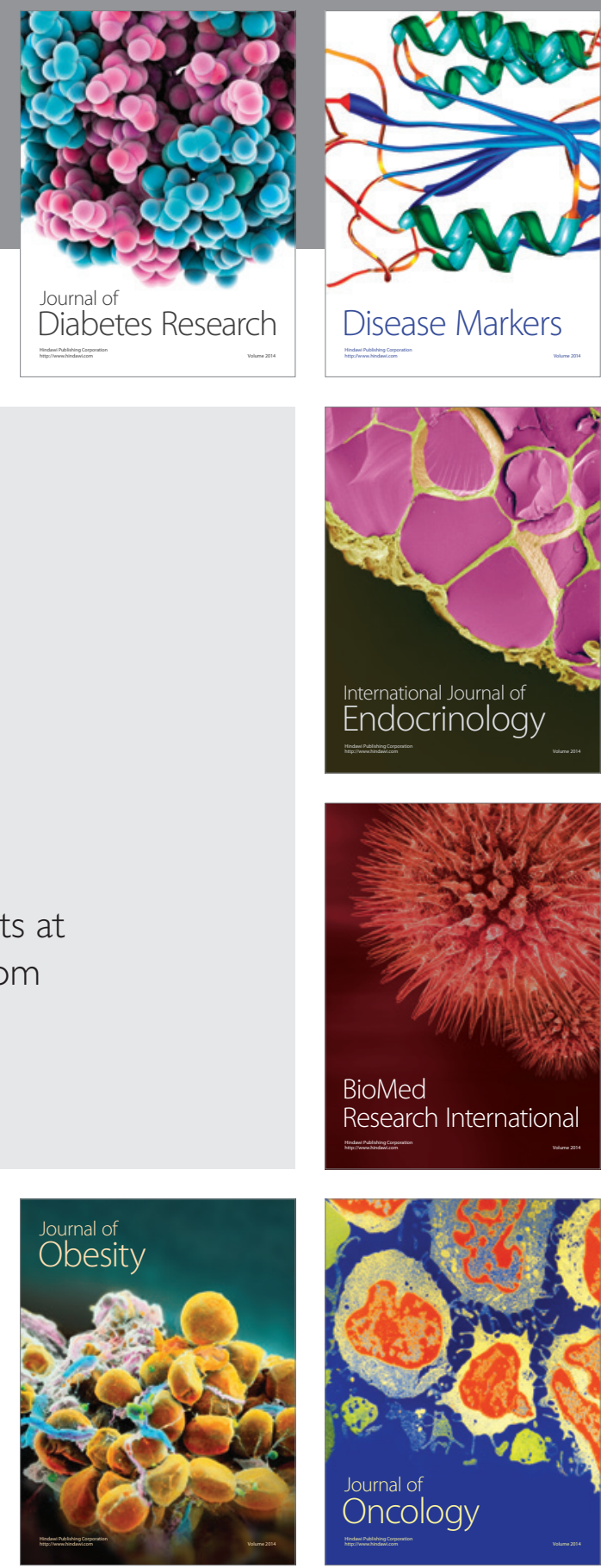

Disease Markers
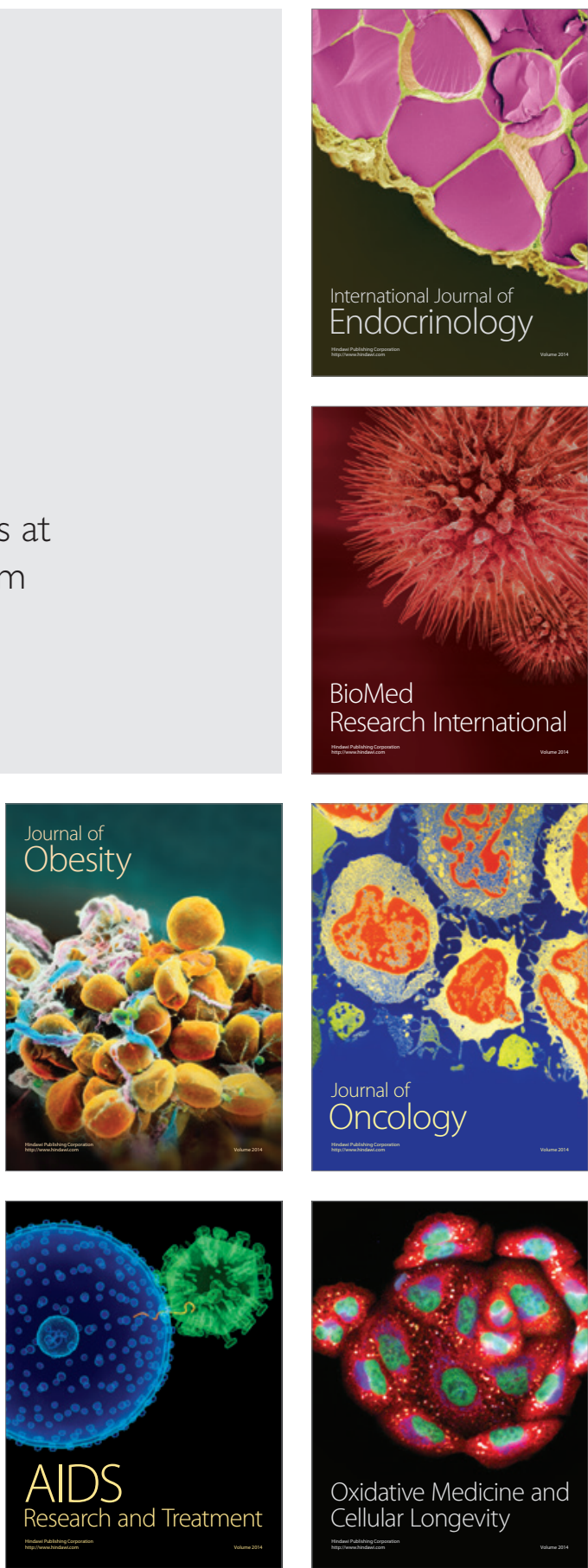